\title{
VIRUS Atlas Austria - COVID-19, labor market and demography at a glance
}

\author{
Florian Korn ${ }^{\text {a, }}$, Karel Kriz ${ }^{\text {b, }}$ Alexander Pucher ${ }^{\mathrm{c}}$ \\ ${ }^{a}$ University of Vienna, florian.korn@univie.ac.at \\ ${ }^{b}$ University of Vienna, karel.kriz@univie.ac.at \\ ${ }^{c}$ University of Vienna, alexander.pucher@univie.ac.at \\ * Corresponding author
}

\begin{abstract}
VIRUS Atlas Austria" (https://map.geo.univie.ac.at/virus_atlas/) is a map-based portal to transport primarily COVID-19 relevant spatio-temporal topics for Austria. In the current version, information on COVID-19 as well as on selected topics of the labor market and demography are integrated. For this purpose, alternative cartographic representations have been developed, to visualize current topics at a glance. The aim of the atlas is to provide the public as well as decision makers with a tool to better assess current risk situations in Austria.
\end{abstract}

Keywords: Thematic maps, Grid maps, COVID-19, Atlas

\section{Introduction}

What is the reproduction number? What is the 7-day incidence in the district where I live? How many elderly people live where? And how is the labor market developing in the different regions? These and other georelevant questions are to be asked and finally answered with the atlas, conveyed with the help of geocommuniaction.

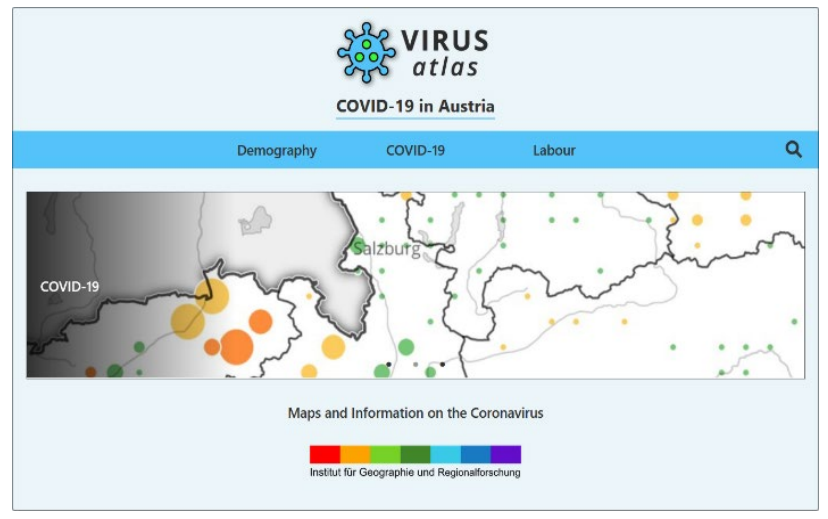

Figure 1: VIRUS atlas - Map collection on current topics with alternative cartographic representations

\subsection{Alternative cartographic representations}

Burgdorf (2009) described the restriction of the choropleth map to relative values as "one of the most important rules in thematic cartography". In contrast to the widespread, area-based, cartographic representations, where values are visualized on administrative units, so-called regular grid representation methods represent an alternative form of representation.

These methods allow a more nuanced perception and interpretability of the same data. A major problem with choropleth maps often is, that they overemphasize large areas with small populations, which in turn cause a very strong visual weighting and can therefore manipulate the viewer's focus. (Speckmann et al. 2010:2). If one relates this value to be represented to the basic quantity, for example the population size, or to the total number of persons with the disease in the area, and then distributes them to predefined weighted population centers, the viewer gets a better spatial impression of the situation. Furthermore, the grid-based representation allows an increased information content through the use of point signatures.

\section{Methodology}

The choropleth map has been an integral part of global cartography since its first appearance in the publication of Charles Dupin. (cf. Skowronnek 2015:2) It is easy to create and most GIS provide simple workflows for creating choropleth maps. Map creation is also in many cases taken out of the hands of professionally trained cartographers and created by individuals with inadequate cartographic skills. The characterized shortcomings of this type of representation (cf. Korn 2019:77) inspired the scientific cartographic communities to develop other techniques, among which is the grid representation, characterized below. 


\subsection{Data sources}

The relevant COVID-19 data provided by the Federal Ministry of Social Affairs, Health, Care and Consumer Protection is fully automated retrieved, processed and imported into a spatial database on a daily basis. In addition, labor market data is provided by the Austrian Labor Market Service on a monthly basis. Population data are obtained from Statistics Austria.

\subsection{Grid representation}

The grid representation is characterized by a grid-based visualization as well as several thematic levels, which are represented by point signatures. The point signatures, which are visualized on a regular grid basis, are in the foreground in order to be able to communicate the facts optimally without distraction and unintentional manipulation. The point signatures can have the classical characteristics in cartography which can be combined in any way, whereby attention must be paid to readability. Therefore, it is recommended to limit the point signatures to three characteristics. (cf. KORN 2019:90)

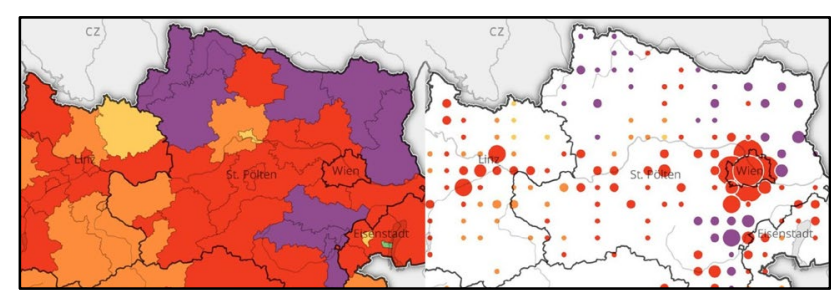

Figure 2: In comparison, the choropleth map (left), only shows the number of SARS-CoV-2 cases per 100,000 inhabitants. The grid map (right), on the other hand, shows the total number of SARS-CoV-2 cases, along with the cases per 100,000 inhabitants based on the district centers on a $10 \mathrm{~km}$ grid.

\subsection{The regular grid}

An essential component is the creation of a regular grid. The grid-based representation is probably the most important characteristic of this map technique. The gridbased representation is primarily intended to offer an alternative where the widely used choropleth map reaches its limits. The data transformation of administrative units by reference points, e.g. population centers, on a raster basis allows an innovative communication approach. The chosen grid size is crucial, considering the map scale and administrative level.

\subsection{Reference points}

Subsequent to the creation of the regular grid, reference points are to be defined. Each area (polygon) is then linked to one or more points. Furthermore, the reference points are used to assign the values of an area to a grid cell. There are various methods for this process, whereby a distinction can be made between the following types of points:
- Thematic points

- Calculated points

- Randomly distributed points

In the present case, thematic reference points are mostly chosen on the basis of population distribution. This is obvious in the case of Austria, since the population is very unevenly distributed.

\subsection{Data matching}

Following the regular grid and reference points preparation, the polygon data is matched to these reference points. Finally, all data within one raster cells is then aggregated. This process is characterized by the following three steps:

- Connection of areas with calculated reference points from regular grid

- Matching of area data onto reference points

- Aggregation of all values in the respective grid cells

\subsection{Point signatures in the center of the grid cell}

As a result, point signatures are placed in the center of each grid cell. In the present case, the signature takes two characteristics. The signature size represents the absolute values, whereas the signature color represents relative values. Additional information could be added through the use of graphic variables, such as shape or signature framing.

\section{Results}

Comparing the traditional mapping method to the potential of the grid representation, it can be seen that the latter, through the grid aggregation as well as the multi-layering has an advantage in terms of the flexibility of the representation of different data compared to the choropleth map.

The first version of the "VIRUS Atlas" was launched in April 2020. Since then, numerous maps have been providing daily information on COVID-19 cases in Austria as well as a selected retrospective view over time. Key demographic data, including age structure, mortality and the situation on the labor market, are also mapped. 


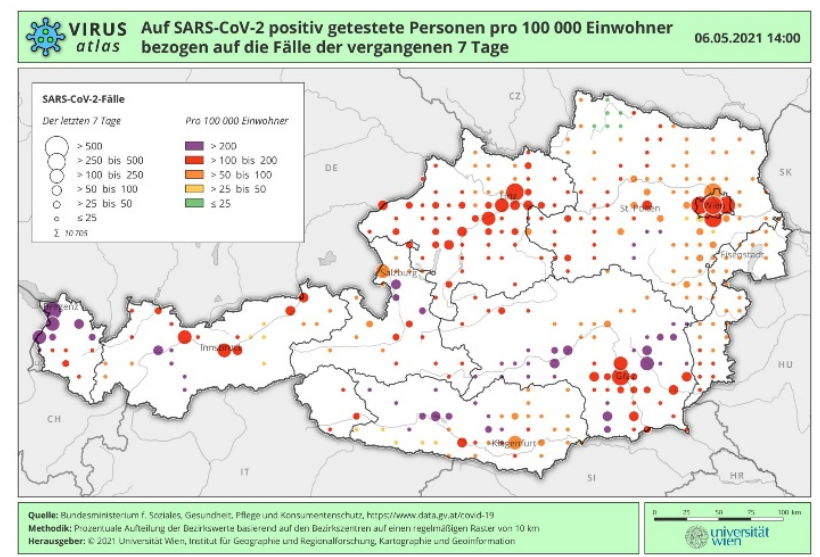

Figure 3: Map showing the total number of SARS-CoV-2 cases within the past 7 days, along with the cases per 100000 inhabitants. The values are determined by percentage distribution of district values based on the district centres on a $10 \mathrm{~km}$ grid.

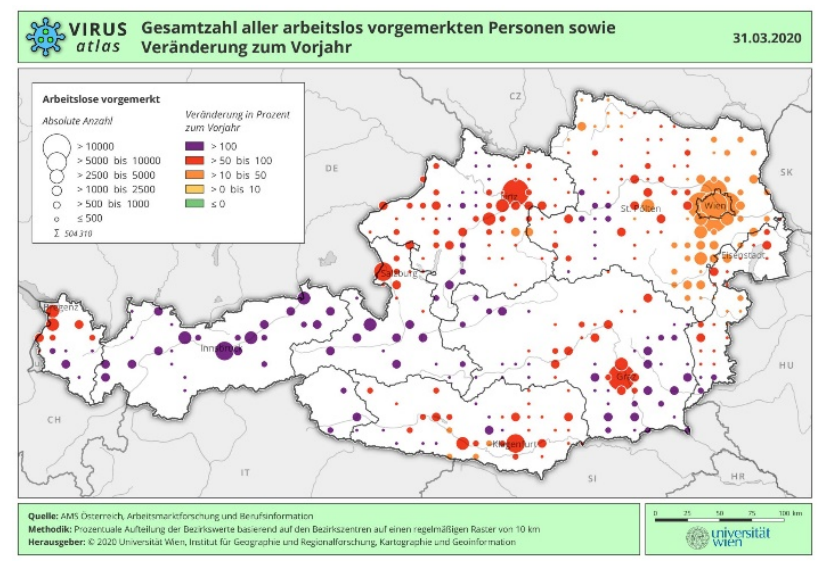

Figure 4: Map showing the number of unemployed people in Austria and the percentage change compared to the previous year. The values are determined by percentage distribution of district values based on the district centres on a $10 \mathrm{~km}$ grid.

\section{Outlook}

In further versions, it is planned to integrate data and maps of other health-related topics. Furthermore, an interactive map viewer will be implemented. However, open questions regarding the availability of data and resources for the further development and operation of the atlas still need to be clarified. Conclusion and experience so far is that the geo-communicative basic principle and the methodical-conceptual implementation of the "VIRUS Atlas" can easily be applied to many topics as well as other spatial entities in a modular way.

\section{References}

Burgdorf M. (2009): Kartogramme: aus der Form geraten oder auf den Punkt gebracht? - In: Informationen zur Raumentwicklung 11 (10), 689-699.
Skowronnek A. (Hrsg.) (2015): Beyond choropleth maps: A review of techniques to visualize quantitative areal geodata; http://alsino.io/beyond-choropleth-maps-a-shortreview-paper, (22.07.2019)

Speckmann B. und Verbeek K. (2010): "Necklace maps," Visualization and Computer Graphics. - In: IEEE Transactions 16(6), 881-889.

Korn F. (2019): „Bestandsaufnahme vorhandener Techniken zur Darstellung von quantitativ, flächenhaften Geodaten und Wege zu einer repräsentativeren Visualisierung und Kommunikation in Form von „Thematic Grid Maps““،. - Wien 